(C) 1996 IEEE. Personal use of this material is permitted. However, permission to reprint/republish this material

for advertising or promotional purposes or for creating new collective works for resale or redistribution to servers

or lists, or to reuse any copyrighted component of this work in other works must be obtained from the IEEE.

\title{
CRYOGENIC THERMOMETRY IN SUPERCONDUCTING ACCELERATORS ${ }^{1}$
}

V.I. Datksov, Joint Institute for Nuclear Research, Dubna, Russia, J.A. Demko ${ }^{2}$, J.G. Weisend, and M. Hentges, Superconducting Super Collider Laboratory, Dallas, Texas, USA

\section{ABSTRACT}

The cryogenic thermometers used in superconducting accelerators must function over a temperature range of $1.5-300 \mathrm{~K}$ in very harsh environments that must be endured for the life of the accelerator. The authors prescribe requirements for cryogenic thermometers in accelerator installations. Thermometer mounting fixtures used in the Joint Institute for Nuclear Research "Nuclotron" (JINR, Dubna, Russia) and the Superconducting Super Collider (SSCL, Dallas, Texas, USA) are described. Experimental results for long-term stability of some cryogenic thermometers and basic recommendations for applications in large superconducting accelerator systems are given.

\section{INTRODUCTION}

Operating modern superconducting (SC) accelerators requires accurate temperature measurements throughout the system. This allows the possibility to (a) monitor and safely control cool-down and warm-up of large mass accelerator magnets, (b) locate high heat leaks and minimize them during adjustment of new accelerator systems, (c) carry out thermal diagnostics of main cryogenic components during accidents, etc. Several peculiarities must be recognized for correctly using cryogenic thermometers in SC accelerators.

(1) Many commercial cryogenic thermometers carry warranties for only 1 or 2 years that may not cover the harsh conditions found in accelerators. Recalibration or verification is typically recommended at frequent intervals.

(2) Accelerators with sizes similar to the Superconducting Super Collider (SSCL) main ring and High Energy Booster System (HEB) have SC magnet lengths of $10 \mathrm{~km}$ and $87 \mathrm{~km}$ respectively, requiring 20-50 thousand cryogenic thermometers. Thermometers are expensive and requires technicians with highly specialized training. Reducing thermometer costs is possible by developing a thermometer in-house based on an inexpensive electronic component, and organizing verification and calibration facilities as was done previously at the ISABELLE project [1], TEVATRON at FNAL [2,3], NUCLOTRON at JINR (Dubna, Russia) [4,5] and others [6,7].

(3) Very harsh conditions exist in superconducting accelerators including (a) high magnetic field up to $6.6 \mathrm{~T}$ [8]; (b) high radiation dosage of about 1000 Mrad for 25 years [8], (c) pressure peak in helium flow during quench up to 2-3 MPa [9], (d) heating of parts (i.e. beam tube) to temperatures of $420-450 \mathrm{~K}$ and cooldown to $4 \mathrm{~K}$ and lower, (e) high voltage electrical insulation 500-3000 V for thermometers in magnet windings, during quench etc.

\section{THERMOMETER REQUIREMENTS}

Some principal requirements for cryogenic thermometers can be established from the requirements. Cryogenic thermometers must (a) Cover wide temperature interval 1.5 - $450 \mathrm{~K}$; (b)

1. This work was supported by the United States Department of Energy under Contract DE-AC04-94AL85000.

2. Present address Sandia National Laboratories, Albuquerque, New Mexico, USA remain stabile for 25 - 50 years better then $0.02-0.03 \mathrm{~K}$ (at 4.2 $\mathrm{K})$; (c) have small, $(<1 \%)$ temperature measurement error in magnetic field $<6 \mathrm{~T}$; (d) be resistant to ionizing radiation (dose $<1000 \mathrm{Mrad}$, temperature error readout $<1 \%$ ); (e) have small $(<1 \%)$ error for pressures up to 3-5 Mpa; (f) have high sensitivity $(\mathrm{dR} / \mathrm{dT})$ particularly in $1.5-10 \mathrm{~K}$ range; $(\mathrm{g})$ have minimal response-time (less than $1 \mathrm{msec}$. at $4.2 \mathrm{~K}$ ); (h) be completely interchangeable; (i) use two wire readout; (j) be resistant to vibration, shocks; (k) have high voltage electrical insulation (500-3000 V); (l) be small; (m) be readily available and inexpensive.

\section{THE “OPTIMAL” THERMOMETER}

Several options for temperature measurement over the interval $1.5 \mathrm{~K}-300 \mathrm{~K}$ using d.c., a.c., and pulse measurement technologies are available. Finding an "optimal" sensor is admittedly an impossible task since no known thermometer has all the best characteristics. The choice of thermometer and measurement system depends on a number of factors, including the required precision, cost, speed of response, sensor size, etc. Rubin and Brandt [12] described the characteristics of some thermometers that can assist in the selection process. Comprehensive cryogenic thermometry reviews are given in $[13,14]$. Anderson reviewed commercial carbon resistors used as cryogenic thermometers for over twenty years [7].

In superconducting accelerators cryogenic thermometers must be resistant to ionizing radiation. S.Scott Courts et all. irradiated several types thermometers at room temperature by gamma source to a level of $10 \mathrm{kGy}$ and neutron+gamma source to a fluence of $8.6 * 10^{13} \mathrm{n} / \mathrm{cm}^{2}$ [15]. In general, diode thermometers are unsuitable for use in either type of radiation environment. For gamma radiation carbon-glass and germanium thermometers performed well for $\mathrm{T}<25 \mathrm{~K}$ and rhodiumiron over $1.4 \mathrm{~K}-300 \mathrm{~K}$. In neutron + gamma radiation the carbon-glass thermometers performed best at lower temperatures and platinum sensors at higher temperatures.

Allen Bradley (AB) carbon resistors have been used worldwide as cryogenic thermometers for more than thirty years because they have high sensitivity, small size, and very low cost. Wehr et. al. irradiated several $\mathrm{AB}$ resistors at $4.6 \mathrm{~K}$ in a reactor up to a dose of $3 * 10^{9} \mathrm{r}$ gammas and thermal and fast $\left(\mathrm{En}=0.1 \mathrm{MeV}\right.$ ) neutrons by 2.5 and $2.0 * 10^{18} \mathrm{n} / \mathrm{cm}^{2}$ respectively [16]. A significant increase of a $110 \mathrm{Ohm} \mathrm{AB}$ resistor from $1500 \mathrm{Ohm}$ up to $3100 \mathrm{Ohm}$ at $4.2 \mathrm{~K}$ (dT about $2.3 \mathrm{~K}$ ) is observed after irradiation. Subsequent annealing to $240 \mathrm{~K}$ lowered dT to $1.6 \mathrm{~K}$. For accurate temperature measurements sufficient radioactivity decay time must be provided.

For the last 18 years at JINR a new type of commercial carbon-ceramic resistor, TVO [4], with $1000 \mathrm{Ohm}$ nominal resistance was tested and is now widely used as a cryogenic thermometer. They possess better long term stability characteristics than $\mathrm{AB}$ resistors as well as a low price. Several TVO carbon-ceramic sensors were irradiated by JINR in a neutron 
reactor at room temperature up to a dose of $8.6 * 10^{9} \mathrm{r} / \mathrm{cm}^{2}$ gammas and fluence $1.3 * 10^{18} \mathrm{n} / \mathrm{cm}^{2}$ fast $(\mathrm{En}=0.1 \mathrm{MeV})$ neutrons [5]. The observed shift in calibration was less than $1 \%$ at $4.2 \mathrm{~K}$. To confirm the JINR data a TVO sensor was included in a cold irradiation experiment conducted by LakeShore Cryotronics and Ohio State University [5]. The data from this experiment show that after gamma doses of $10 \mathrm{kGy}$ at $4.2 \mathrm{~K}$ the shift in calibration for TVO is less than $1 \%$.

\section{LONG-TERM STABILITY}

AB $0.125 \mathrm{~W}, 100 \mathrm{ohm}$ nominal resistors have been used for 10 years in large cryogenic systems at JINR. The temperature resistance $(\mathrm{T}(\mathrm{R}))$ characteristics showed significant changes (>2\%) after 1-3 years particularly when mounted on cryogenic surfaces in a vacuum. After several cooldowns to $4.2 \mathrm{~K}$ over 6 months some $\mathrm{AB}$ resistors increased resistance up to $105 \mathrm{Ohms}$ with a room temperature readout dT of 5-20 K. Periodically (3 times per year) $10 \mathrm{TVO}$ are tested at JINR shows high level of stability with $<15 \mathrm{mK}$ shift at $4.2 \mathrm{~K}$ over 14 years[4].

At the SSCL a calibration and test facility was organized and supported the Accelerator Systems String Test (ASST). Significant changes in AB thermometer calibration was observed in thermometers which were calibrated and mounted at FNAL on support-tubes of 323 dipole magnet. During preparations for ASST Run \#3 the deviation from known room temperature of $\mathrm{AB}$ readout was -15 to $+67 \mathrm{~K}$. Our SSCL research with several of $100 \mathrm{Ohm} \mathrm{AB}$ showed that after 30 thermal-cycles the $T(R)$ characteristics shift at $4.19 \mathrm{~K}$ is $8-20$ $\mathrm{mK}$, at $77.2 \mathrm{~K}$ is $0.6-3.2 \mathrm{~K}$. Instability in $\mathrm{AB}$ carbon resistors has been the subject of a number of reports since being used for cryogenic thermometry $[7,17]$. At the same time TVO resistor testing showed a shift in $\mathrm{T}(\mathrm{R})$ after 30 thermal-cycles at $4.19 \mathrm{~K}$ of $4-6 \mathrm{mK}$, and at $77.2 \mathrm{~K}$ of $0.15-0.3 \mathrm{~K}$.

Three runs of the ASST showed some problems with capsuled thermometers mounted in the liquid helium stream in dipole magnets. During Run \#1 after a quench a carbon-glass CGR1-1000 thermometer suddenly changed calibration at 4.19 $\mathrm{K}$ dT of $72 \mathrm{mK}$ and at $77.2 \mathrm{~K}$ dT of $7.45 \mathrm{~K}$. During later testing and detailed analysis a gas leak was observed from thermometer capsule. The quench high pressure pulse of 15-20 Bar probably damaged the capsuled thermometer seal. During ASST Run\#3 (17-Sept-93) the magnet string was filled with liquid helium without flow at a pressure of 1.22 Bar corresponding to a saturation temperature of $4.42 \mathrm{~K}$. The $16 \mathrm{CGR}$ thermometers in the cold mass showed different temperatures: 8 thermometers differed from $4.42 \mathrm{~K}$ by $<10 \mathrm{mK}$, and 8 others differed from 0.1 - to a few K indicating possible damage. Differences were observed in readings of intact and damaged thermometers when self-heated by high measurement current. Also during a second test of used capsuled thermometers after thermal cycling significant ac noise was observed below 8-9 K.

\section{APPLYING CRYOGENIC THERMOMETERS}

Even with quality thermometry high accuracy measurements are difficult without experience in thermometer application. Many laboratories around the world invest in small installations to gain experience with cryogenic thermometers i.e. mounting, gluing wires, shielding measurement places, etc.
An improperly mounted or heat sunk thermometer will read a temperature contribution from the electrical leads. Careful planning with regard to the application must be performed early on. It is recommended to have an accurate thermal model of the sensor placement and a method for in-situ verification of the thermometer. A thermal model can provide a necessary assessment of the accuracy of the temperature measurement. As an example of a thermal model, assume a thermometer has a resistance of $10 \mathrm{kohms}$ at $4.2 \mathrm{~K}$ and is mounted on a surface at $4.2 \mathrm{~K}$. There are four \#32 AWG leads (total cross section of $0.051 \mathrm{~mm}^{2}$ ) 1 meter long going to the thermometer in a vacuum from a $300 \mathrm{~K}$ connector. The sensing current is 10 micro Amperes. Using a numerical method of lines (NUMOL) solution as discussed for power leads in [10], the dependence of the error on contact between the sensor and the measurement surface can be evaluated. The error due to contact for coefficients of $10000 \mathrm{~W} / \mathrm{cm}^{2} / \mathrm{K}$ and $100 \mathrm{~W} / \mathrm{cm}^{2} / \mathrm{K}$ based on lead cross section are $0.0015 \mathrm{~K}$ and $0.1516 \mathrm{~K}$ respectively.

The determination of the measurement uncertainty must be performed similar to [11] and used to guide placement and mounting of thermometers. Results in [11] for the ASST show that the $4 \mathrm{~K}$ heat leak across a single dipole magnet 15 meters long, must be determined from small temperature differences of $10-50 \mathrm{mK}$. High accuracy temperature sensors and mounting techniques are required to minimize the uncertainty in heat leak measurements.

In addition to temperature errors from improper mounting techniques, sensors can drift from their initial calibration. It is desirable to have a method of checking / verifying thermometer calibration as installed (in situ) for periodic evaluation of the sensor and the data acquisition system. Redundant thermometers are frequently applied to accomplish this. It may be possible to re-calibrate some sensors with the aid of thermal modeling of the system performance used with measurements from temporary precision instrumentation over large sections (say 10 or more superconducting magnets) of an accelerator.

Cryogenic thermometer mounting may be performed by personnel with limited experience. One remedy is to develop simple thermometer mounting fixtures. For the NUCLOTRON indirect temperature measurement techniques were developed to avoid installing thermometers in helium flow lines eliminating many vacuum feedthroughs reducing the possibility of leaks. The mounting fixture for a helium tube is shown in Figure 1. Twisted wires (8) from the hermetic connector at $300 \mathrm{~K}$ sink feed into the screw-thermal anchor (9) on copper plate (6), which is soldered on He tube (7). The screw-thermometer (2) measures the temperature of helium tube (7) with a $1-2 \%$ error over $4-300 \mathrm{~K}$. The thermometer (3) is a TVO and bifilar winding (4) using a special technique for mounting in the screw (2) covered by a copper cover (1). All surfaces are polished and nickel plated. About 700 of these are installed in the NUCLOTRON on helium tubes in vacuum space and have performed well for over 5 years.

For the SSCL a different thermometer mounting fixture using TVO sensors was designed and installed in about 25 locations in the ASST. The fixture for a helium tube in the 
interconnect between dipole magnets is shown in Figure 2. The thermometer (1) and thermal anchor (2) are mounted in holes of a stainless steel plate (3) using thermal-conducting grease (9). The fixture is clamped on the helium tube (8) with a hose clamp (5) and covered by multilayer insulation (6). During ASST Run \#3 this fixture measured the tube temperature to within $0.01 \mathrm{~K}$ at $4.4 \mathrm{~K}$ from another carbon-glass thermometer mounted nearby in the magnet cold mass in the liquid helium stream. These were also installed on the $20 \mathrm{~K}$ and $80 \mathrm{~K}$ shields.

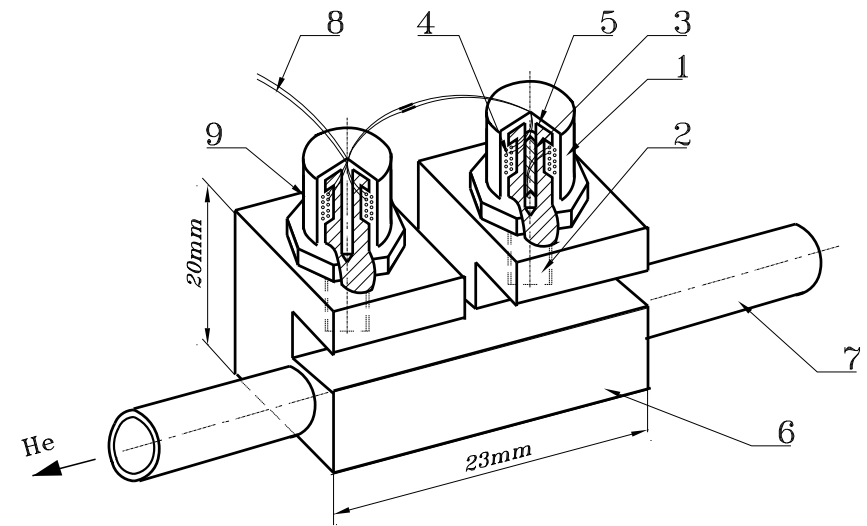

Figure 1: JINR Thermometer mounting fixture
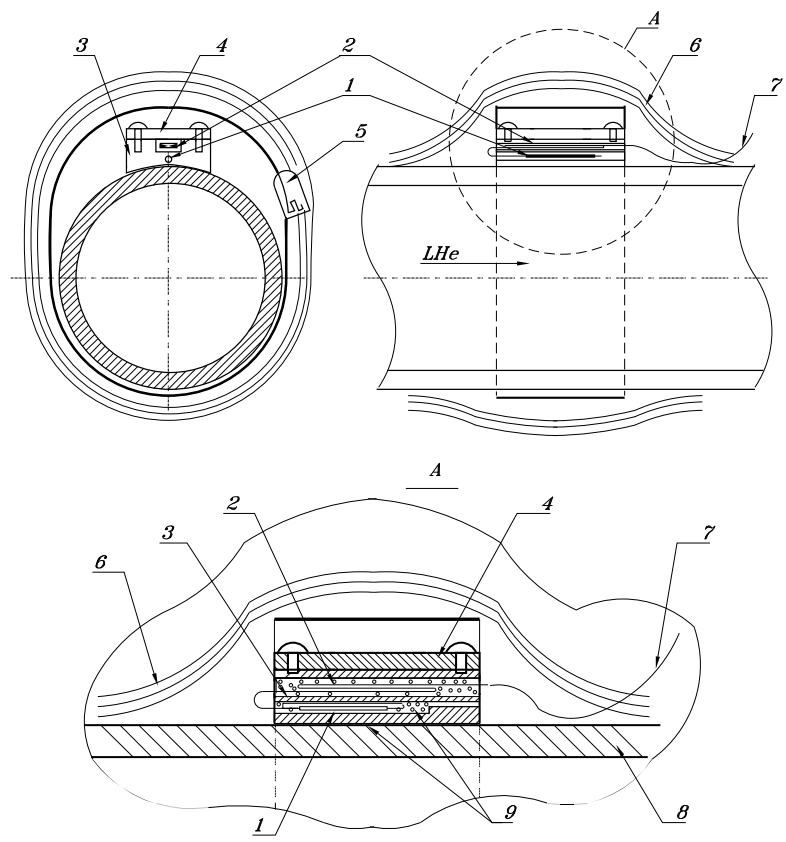

Figure 2: SSC Thermometer mounting fixture

\section{CONCLUSIONS}

For large superconducting accelerators it is necessary to have a small group of highly skilled, professional people and a calibration and test facility. This group should design special mounting fixtures, do full input control of all thermometers in real conditions, test and analyze damaged thermometers, model and predict future characteristics. Thermometers for superconducting particle accelerators must be able to withstand very severe conditions for the life of the accelerator. In-situ calibration/verification techniques using advanced thermal modeling of large sections of particle accelerators can be used to reduce the need for thermometer replacement.

\section{REFERENCES}

[1] J. Sondericker, "Production and use of high grade silicon diode temperature sensors", Advances in Cryogenic Engineering, vol.27, 1982

[2] M. Kuchnir, "Automatic measurements of heat load," Advances in Cryogenic Engineering, vol.27,1982.

[3] M. Kuchnir, "Pulsed current resistance thermometry," Advances in Cryogenic Engineering, vol.29, 1984.

[4] V.I. Datskov, L.V. Petrova, G.P. Tsvineva. "Cryogenic thermometers based on the TVO type resistors and their application", (in Russian). Communication of the JINR, \#P8-87-604, Dubna, 1987.

[5] V.I. Datskov, J.G. Weisend II, "Characteristics of Russian carbon resistance (TVO) cryogenic thermometers," Cryogenics, vol.34, ICEC Supplement, 1994.

[6] E.M.W. Leung, R.D. Kephart, A.S. Ito and R.W. Fast, "The superconducting Chicago cyclotron magnet," Advances in Cryogenic Engineering, vol.27,1982.

[7] A.C. Anderson, "Carbon resistance thermometry," Temperature, Its. Measurement and Control in Science and Industry, vol.4,Instrument Society of America, Pittsburgh, 1972

[8] R. Johnson and N. Mokhov, "Beam loss monitor system for the SSC". SSCL-Preprint-523, Dallas (October, 1993).

[9] W. Burgett, et. al. Cryogenic characteristics of the SSC Accelerator System String Test," Supercollider 5, 1994

[10] K. Stifle and J. Demko, "Evaluation of the Accelerator System String Test (ASST) Heat Leak Measurements and Their Uncertainties," SSCL Note SSCL-N-871, 1994.

[11] J. Demko and V. Datskov, "Operation and Control of the Helium Cooled Power Leads for the Superconducting Super Collider", SSCL Note SSCL-N-870, 1994.

[12] L.G. Rubin, B.L. Brandt and H.H. Sample, "Some practical solutions to measurement problems encountered at low temperatures and high magnetic fields," Advances in Cryogenic Engineering, Vol.31, 1986

[13] L.G. Rubin, B.L. Brandt and H.H. Sample, "Cryogenic thermometry: a review of recent progress, II," Cryogenics, vol.22, 1982.

[14] S.Scott Courts, D.Scott Holmes, Philip R. Swinehart and Brad C.Dodrill, Applications of Cryogenic Technology, vol.10, 1991.

[15] S. Scott Courts, D. Scott Holmes, and Philip R. Swinehart, Lake Shore Cryotronics, Personal Communication, 1994.

[16] G. Wehr, G. Sieber, and K. Boning, "Carbon resistors as low temperature sensors in low temperature reactor irradiation experiments," Cryogenics, 1977.

[17] L.M. Besley, "Stability of some cryogenic carbon resistance thermometers," Rev. Sci. Instrum., 54 (9), 1983.

\section{ACKNOWLEDGMENTS}

The authors gratefully acknowledge the assistance of L. Petrova and staff of the JINR calibration laboratory; M. Kuchnir, W. Boroski of Fermilab; J.Swartz, D.Holmes and S.Courts of LakeShore Cryotronics; M. Levin, S.Augustinowicz, L. Richards, Earnest Williams, B. Fietz, M. McAshan formerly at the SSCL, and J Spieckerman of MarketTech International. 